$\mathrm{UW} / \mathrm{PT}$ 00-04

\title{
Electroweak Bubble Wall Friction: Analytic Results
}

\author{
Guy D. Moore \\ Department of Physics, University of Washington, Seattle WA 98195-1560 USA
}

(January 2000)

\begin{abstract}
We present an entirely analytic, leading log order determination of the friction an electroweak bubble wall feels during a first order electroweak phase transition. The friction is dominated by $W$ bosons, and gives a wall velocity parametrically $\sim \alpha_{w}$, and numerically small, $\sim .01-0.1$ depending on the Higgs mass.
\end{abstract}

\section{INTRODUCTION}

Electroweak baryogenesis is the name for the production of the baryon number asymmetry of the universe at the electroweak epoch. It is possible in extensions of the standard model where the three famous Sakharov conditions can be met:

1. Baryon number violation is efficient,

2. The discrete symmetries $\mathrm{C}$ and $\mathrm{CP}$ are violated, and

3. There is a departure from equilibrium, coincident with baryon number violation turning off.

All three of these criteria potentially exist in the standard model. Baryon number violation is not only present, but efficient [1,2]. The expansion of the universe provides departure from equilibrium. And of course, $\mathrm{C}$ and $\mathrm{CP}$ are known not to be true symmetries.

Whether or not electroweak baryogenesis can explain the size of the observed baryon number of the universe, which is about [3]

$$
\frac{\text { baryons }}{\text { entropy }} \simeq(2-7) \times 10^{-11}
$$

is a more detailed question. In the minimal standard model, both the available $\mathrm{CP}$ violation [4], and the departure from equilibrium [5], appear to be grossly insufficient. However the question is quite open in extensions of the standard model.

Baryogenesis at a first order electroweak phase transition is a complicated process, and requires understanding several things. First, we must be able to compute the strength of the phase transition. The tools for doing this are now well developed [5].6]. Next, we must be able to compute the efficiency of baryon number violation; here the tools are also well developed [7] [12. Finally one must be able to compute the microscopic dynamics of baryon 
number carrying excitations in the presence of an electroweak phase interface, henceforth called the "bubble wall" [13]. This problem is not well under control, but the papers quoted show that the results strongly depend on another factor, which is the velocity of propagation of the bubble wall. This paper will discuss the computation of this bubble wall velocity, which is important for baryogenesis, and is also interesting as an example where such a dynamical quantity can be computed from first principles.

A fairly substantial literature already exists on the electroweak bubble wall velocity. Since a bubble wall liberates latent heat as it propagates, hydrodynamic considerations are potentially important. Hydrodynamic considerations appear in [14] [18]. The conclusion is that, if the friction on the bubble wall is small, then the hydrodynamics are important; but if the bubble friction is large, so the wall velocity is small, then all that matters is the general rise in temperature from the bubbles in aggregate as the transition proceeds.

There is also a literature on the friction the bubble wall feels [19 25]. The paper of Khlebnikov [22] shows how the friction is related to the self-energy of the zero mode of the bubble wall. That is, the friction arises from the back-reaction on the wall of the disturbance from equilibrium of excitations, induced by the motion of the wall. Most of the papers quoted study this back reaction by treating the excitations with kinetic theory. The exception is [25], where it is argued that infrared $\mathrm{SU}(2)$ gauge field and Higgs field excitations are most important, and that it is more appropriate to treat them as classical fields, which can be done nonperturbatively on the lattice.

Both the kinetic descriptions, and classical nonperturbative treatment, missed one important piece of physics, however, which as we will see leads them to be incorrect parametrically. That is, they miss the physics of screening and Landau damping, which dominates the dynamics of infrared gauge fields. The importance of this physics has been pointed out by Arnold, Son, and Yaffe, [7,26.227, in the context of determining the baryon number violation rate, and has been further discussed in [9,28,29, 12]. The central result is that the $\mathrm{SU}(2)$ gauge field $A$, instead of evolving under (classical) equations of motion of the form (in temporal gauge, ignoring nonlinearities)

$$
\frac{d^{2} A}{d t^{2}}=-\left(k^{2}+m^{2}\right) A,
$$

with $m^{2}=\left(g^{2} / 4\right) \phi^{2}$ the mass squared induced by a Higgs condensate, instead undergoes overdamped evolution,

$$
\frac{\pi m_{\mathrm{D}}^{2}}{4 k} \frac{d A}{d t}=-\left(k^{2}+m^{2}\right) A+\text { noise },
$$

with $m_{\mathrm{D}}^{2}$ the Debye mass squared, $m_{\mathrm{D}}^{2}=(11 / 6) g^{2} T^{2}$ in the standard model. In this letter we will see what consequences this has for the kinetic description of the bubble wall friction.

\section{INGREDIENTS}

For concreteness we will work here with the minimal standard model, even though baryogenesis in that model is ruled out. Since only the gauge fields are overdamped, and since only the $\mathrm{SU}(2)$ and $\mathrm{U}(1)$ fields have large interactions with the Higgs condensate, the extension to models with more fields, such as the minimal supersymmetric standard model, 
should be straightforward. From here on we will also neglect the $\mathrm{U}(1)$ field, which is the same as taking $g^{\prime} \ll g$. This is probably a reasonable approximation. Going beyond it would increase the bookkeeping but would not make our treatment substantially more difficult.

This work will be strictly analytic and strictly based on parametric expansions in formally small quantities. In order to obtain an electroweak phase transition of a strength which can be analyzed perturbatively, we will take

$$
g^{3} \ll \lambda \ll g^{2} / \log (1 / g),
$$

where $\lambda$ is the Higgs self-coupling in the same normalization as used in 20. As we see in a moment, the gauge field condensate is parametrically $\phi_{0} \sim g^{3} T / \lambda$, so the induced gauge field masses are $g \phi_{0} / 2 \sim g^{4} T / \lambda$. The longitudinal gauge fields have a Debye mass $\sim g T \gg g^{4} T / \lambda$ and can be neglected [20]; the one loop effective potential is then approximately [20]

$$
V_{1 \text { loop }}(\phi)=\frac{m^{2}(T)}{2} \phi^{2}--\frac{g^{3} T}{16 \pi} \phi^{3}+\frac{\lambda}{4} \phi^{4} .
$$

A broken minimum exists if $d V / d \phi=0$ at some nonzero $\phi_{0}$. The broken minimum is degenerate with the minimum at $\phi=0$ at

$$
m^{2}\left(T_{\mathrm{eq}}\right)=\frac{g^{6} T^{2}}{128 \pi^{2} \lambda},
$$

and the value of $\phi_{0}$ is

$$
\phi_{0}\left(T_{\mathrm{eq}}\right)=\frac{g^{3} T}{8 \pi \lambda} .
$$

The $\phi$ profile of the electroweak bubble wall at $T_{\text {eq }}$ is given at leading order by

$$
\phi(z)=\frac{\phi_{0}}{2}\left[1+\tanh \frac{z}{L}\right], \quad L=\frac{2}{m\left(T_{\mathrm{eq}}\right)} .
$$

Here $z$ is a space coordinate orthogonal to the bubble wall.

The gauge field mass times the wall width,

$$
L m_{W}=L \frac{g \phi}{2}=\frac{16 \pi \sqrt{2 \lambda}}{g^{3} T} \frac{g^{4} T}{16 \pi \lambda} \frac{\phi}{\phi_{0}}=\frac{\phi}{\phi_{0}} \sqrt{\frac{2 g^{2}}{\lambda}} \gg 1,
$$

is large, and it is therefore possible to treat gauge field excitations, in the broken phase and inside the bubble wall, with kinetic theory. The kinetic theory description, which amounts to taking the Higgs field background as approximately homogeneous and expanding in its gradients, breaks down at the small $z$ (symmetric phase) edge of the bubble wall, when $\left(\phi / \phi_{0}\right) \sim \sqrt{\lambda / g^{2}}$. In fact, since $\lambda=\left(g^{2} / 8\right)$ is the condition for $m_{H}=m_{W}$, the kinetic description works fairly well at remarkably large Higgs masses.

All of the equations above are valid at leading order in $\lambda / g^{2}$ or $g^{3} / \lambda$. when the former breaks down, higher loop corrections and corrections from Higgs loops (neglected here) become important. When the latter breaks down, the longitudinal gauge fields become important. We also need $g^{3} \ll \lambda$ to ensure that the relevant gauge fields will be overdamped. 


\section{FRICTION}

The friction on an electroweak bubble wall is defined as the excess pressure on the wall (directed towards the broken phase), over the equilibrium value;

$$
\text { friction }=P-P_{\text {eq }}=P+V(\phi=0)-V\left(\phi_{0}\right) \equiv P+\Delta V \text {. }
$$

We expect the friction to depend on the bubble wall velocity, with the condition $P=0$ determining the steady state bubble wall velocity $v_{w}$, which is what we want to know. We can define a linear response friction coefficient $\eta$ as a limit

$$
\eta \equiv \lim _{\Delta V \rightarrow 0} \frac{\Delta V}{v_{w}}=\frac{\text { steady state friction }}{v_{w}}
$$

This is what we want to determine.

The friction on the bubble wall depends on the departure from equilibrium of the plasma excitations inside the bubble wall. In a kinetic theory description, the excitations are described by population functions $f(k, x)$. We write them as an equilibrium part $f_{0}$,

$$
f_{0}=\frac{1}{\exp (E / T) \pm 1}, \quad E=\sqrt{k^{2}+m^{2}(x)}
$$

with + for fermions and - for bosons, which is the case we will care about, plus a departure from equilibrium $\delta f$. The friction a bubble wall feels is, in the kinetic description [19]21,24],

$$
\text { friction }=\int_{-\infty}^{\infty} d z \sum_{\mathrm{DOF}} \int \frac{d^{3} k}{(2 \pi)^{3}} \frac{d \phi}{d z} \frac{d m^{2}}{d \phi} \frac{d E}{d m^{2}} \delta f,
$$

where the sum is over degrees of freedom which get a mass from the Higgs field. This expression has a clear intuitive meaning; it is the sum over excess particles of $(d E / d z)$, the force the wall exerts on them. It remains to determine $\delta f$ and evaluate the integral.

The friction will be dominated by the gauge boson contribution. The contribution from Higgs bosons is smaller because their $d m^{2} / d \phi$ is smaller by $\lambda / g^{2}$, and because their evolution is not overdamped. The fermionic contributions, such as that from the top quark, are smaller because Fermi-Dirac statistics lack the infrared divergence of Bose-Einstein statistics, so their $\delta f$ has much weaker infrared behavior. The parametric argument appears in [25], and a reasonable estimate of friction from top quarks appears in [24]; it proves numerically smaller than what we find below. The case where there is a light scalar top is more difficult and we do not consider it; for the MSSM the friction we find should be viewed as a lower bound rather than a tight estimate.

As discussed above, the gauge fields undergo overdamped evolution given by Eq. (1.3). Since $f \propto A^{2}$, the equation for $f$ is

$$
\frac{\pi m_{\mathrm{D}}^{2}}{8 k} \frac{d f}{d t}=-E^{2} f+\text { noise }
$$

\footnotetext{
${ }^{1}$ A derivation of sorts can be found in [24, but the expression is implicit in the earlier references as well.
} 
where the noise is of the right size to ensure that, for $m^{2}$ time independent, $f$ will approach $f_{0}$; so averaging over the noise,

$$
\left(k^{2}+m^{2}\right) f+\text { noise } \rightarrow E^{2} \delta f .
$$

Also, $d f / d t=d f_{0} / d t+d(\delta f) / d t$. At small $v_{w}$, the limit we are interested in, $\delta f \ll f_{0}$, and $d(\delta f) / d t$ may be dropped. Further,

$$
\frac{d f_{0}}{d t}=\frac{d \phi}{d t} \frac{d m^{2}}{d \phi} \frac{d E}{d m^{2}} \frac{d f_{0}}{d E}=-v_{w} \frac{d \phi}{d z} \frac{d m^{2}}{d \phi} \frac{1}{2 E T} f_{0}\left(1+f_{0}\right)
$$

Therefore, the departure from equilibrium of a gauge boson degree of freedom is

$$
\delta f=\frac{\pi m_{\mathrm{D}}^{2} v_{w}}{16 k E^{3} T} f_{0}\left(1+f_{0}\right) \frac{d \phi}{d z} \frac{d m_{W}^{2}}{d \phi} .
$$

Note that transport plays no role in setting $\delta f$; this is because the gauge fields are overdamped. Substituting this into Eq. (3.4), and noting that there are 6 species of transverse $W$ bosons (3 flavors times 2 spins), gives

$$
\text { friction }=\frac{6 \pi v_{w} m_{\mathrm{D}}^{2}}{8} \int_{-\infty}^{\infty} d z\left(\frac{d \phi}{d z} \frac{d m_{W}^{2}}{d \phi}\right)^{2} \int \frac{d^{3} k}{(2 \pi)^{3}} \frac{f_{0}\left(1+f_{0}\right)}{4 k E^{4} T} .
$$

Since $f_{0}$ is monotonically decreasing, the momentum integral is infrared dominated, cut off by the nonvanishing $W$ boson mass. Therefore it is appropriate to make the approximation, valid in the infrared, that $f_{0} \simeq 1+f_{0} \simeq T / E$, and evaluate the integral;

$$
\int \frac{d^{3} k}{(2 \pi)^{3}} \frac{f_{0}\left(1+f_{0}\right)}{4 k E^{4} T} \simeq \int_{0}^{\infty} \frac{k^{2} d k}{2 \pi^{2}} \frac{T}{4 k E^{6}}=\frac{T}{16 \pi^{2}} \int_{m_{W}^{2}}^{\infty} \frac{d\left(E^{2}\right)}{E^{6}}=\frac{T}{32 \pi^{2} m_{W}^{4}} .
$$

The friction, using $m_{W}=g \phi / 2$, is then

$$
\text { friction }=v_{w} \frac{3 m_{\mathrm{D}}^{2} T}{32 \pi} \int_{-\infty}^{\infty} d z\left(\frac{d \phi}{d z}\right)^{2} \frac{1}{\phi^{2}} .
$$

Writing $(d \phi / d z) d z=d \phi$, and using Eq. (2.5) to write

$$
\frac{d \phi}{d z}=\frac{2 \phi\left(\phi_{0}-\phi\right)}{L \phi_{0}}
$$

Eq. (3.11) now gives

$$
\eta=\frac{3 m_{\mathrm{D}}^{2} T}{16 \pi L} \times\left(\int_{0}^{\phi_{0}} \frac{\left(\phi_{0}-\phi\right) d \phi}{\phi_{0} \phi}=\int_{0}^{1} \frac{(1-x) d x}{x}\right) .
$$

There is a log divergence arising from the symmetric phase side of the bubble wall. The log will be cut off where the first approximation used to derive Eq. (3.11) breaks down. The perturbative expansion breaks down when $m_{W} \sim g^{2} T$, or $\phi \sim g T$, which is at $\left(\phi / \phi_{0}\right)=$ $\left(\lambda / g^{2}\right)$. The kinetic theory description breaks down at $\left(\phi / \phi_{0}\right)=\sqrt{\lambda / g^{2}}$, see Eq. (2.6); this occurs first. 
Since the degrees of freedom which dominate the friction are those with $k \sim m$, when $m$ drops below $1 / L$, it is no longer appropriate to treat the particles as seeing a slowly varying wall. Such degrees of freedom see a wall which is sharper than their wavelength can resolve. For those degrees of freedom with $k L \gg 1$, we find the friction scales as $1 / L$. This must go over to an $L$ independent value for wavelengths which cannot resolve the thickness of the wall, which means that their contribution is less than the kinetic theory estimate. Hence the log is cut off at $\left(\phi / \phi_{0}\right) \sim \sqrt{\lambda / g^{2}}$, the contribution from very infrared degrees of freedom is subdominant. Hence the friction we determine is

$$
\eta=\frac{3 m_{\mathrm{D}}^{2} T}{16 \pi L}\left(\log \left(m_{W} L\right)+O(1)\right)=\frac{3}{16 \sqrt{2}} \frac{m_{D}^{2}}{g^{2} T^{2}} \frac{g}{\sqrt{\lambda}} \alpha_{w}^{2} T^{4}\left(\log \frac{g}{\sqrt{\lambda}}+O(1)\right) .
$$

The first expression makes no assumptions about the effective potential or wall thickness and should be valid in extensions as well as the standard model.

Now we comment on the parametric form of $\eta$. Taking $\lambda \sim g^{2}$ and neglecting logs, the friction coefficient is $\eta \propto \alpha_{w}^{2} T^{4}$. We can guess this on dimensional grounds by noting that

$$
[\eta]=\left[\frac{\text { pressure }}{\text { velocity }}\right]=\left[\frac{\text { energy } \times \text { time }}{\text { length }}{ }^{4}\right],
$$

and that $\eta$ arises from infrared gauge field physics. Such physics has a natural energy scale $\sim T$, a natural length scale $\sim 1 / \alpha_{w} T$, and a natural time scale $\sim 1 /\left(\alpha_{w}^{2} T\right)$ [7]; so on dimensional grounds we should have anticipated $\eta \sim \alpha_{w}^{2} T^{4}$. This is to be contrasted with the pressure driving the bubble wall, which by the same parametric estimates must be

$$
[P]=\left[\frac{\text { energy }}{\text { length }^{3}}\right], \quad P \sim \alpha_{w}^{3} T^{4} .
$$

Hence, the bubble wall velocity is parametrically $v_{w} \sim \alpha_{w}$.

\section{BÖDEKER'S EFFECTIVE THEORY}

The reason we considered the case $\lambda \ll g^{2} / \log (1 / g)$, rather than $\lambda \ll g^{2}$, is because in the parametric regime

$$
\frac{g^{2}}{\log (1 / g)} \ll \lambda \ll g^{2}
$$

The gauge bosons with $m \sim g \phi_{0} / 2$ do not obey Eq. (1.3); instead Bödeker's effective theory is applicable [9];

$$
\sigma \frac{d A}{d t}=-E^{2} A+\text { noise }, \quad \sigma=\frac{2 \pi m_{\mathrm{D}}^{2}}{3 g^{2} T \log (1 / g)},
$$

up to corrections suppressed by $\log (1 / g)$. In this case the derivation proceeds analogously, but the behavior is slightly less infrared dominated, and no log occurs in the $\phi$ integral. The final expression is

$$
\eta=\frac{1}{256 \sqrt{2} \log (1 / g)}\left(\frac{g}{\sqrt{\lambda}}\right)^{3}\left(\frac{m_{D}^{2}}{g^{2} T^{2}}\right) \alpha_{w}^{2} T^{4}
$$


This result is in parametric agreement with what we found above. It is only applicable in an extremely narrow parametric range, and receives corrections suppressed only by $\left(\lambda / g^{2}\right)$ (from the loop expansion) and $g^{2} \log (1 / g) / \lambda$ (from the breakdown of Bödeker's effective theory).

\section{CONCLUSION: VALUE OF THE WALL VELOCITY}

Since we only have the friction at leading log, which means with at least a factor of 2 error, we will do with a fairly crude estimate of the pressure which drives the bubble. In references [20,21] the nucleation temperature is estimated as occurring at $m^{2}\left(T_{\text {nuc }}\right)=0.8 m^{2}\left(T_{\text {eq }}\right)$. For this value, using Eqs. (2.2) and (2.3), the pressure driving the bubble wall is approximately $P=.94 \times\left(g^{6} / 1024 \pi \lambda^{3}\right) \alpha_{w}^{3} T^{4}$, and the wall velocity is about

$$
v_{w}=\frac{P}{\eta} \simeq .0012\left(\frac{g}{\sqrt{\lambda}}\right)^{5} \frac{\alpha_{w}}{\log (g / \sqrt{\lambda})},
$$

which, for $m_{H} \simeq m_{W}$ or $\lambda=g^{2} / 8$, and estimating $\log (g / \sqrt{\lambda}) \simeq 1$, is $v_{w} \simeq \alpha_{w} / 4<0.01$. For $\lambda / g^{2} \simeq .04$, as required to give a sufficiently strong phase transition, $v_{w} \sim 0.1$. The numerical value for the bubble wall velocity is expected to be small. The numerical estimate from the result determined using Bödeker's effective theory is similar; $v_{w} \sim 0.2$ for $\lambda / g^{2}=.04$. Neither estimate is very reliable because the constant under the log has not been determined; but the conclusion $v_{w} \ll 1$ is clear.

The friction we find is larger, and the value of $v_{w}$ smaller, than in previous literature. In particular we find a much larger friction than the numerical results of [25] indicate. This is partly because there we did not include hard thermal loop effects for the gauge fields, but overly aggressive data fitting may have contributed. It would be interesting to make a new numerical analysis using the techniques developed in [11].

We should also briefly comment on how the result may change in extensions to the standard model and beyond leading order in $v_{w}$. Beyond first order in $v_{w}$, a few effects become important. We may not be able to neglect $\delta f$ next to $f_{0}$ in getting Eq. (3.8); $\delta f$, and the friction, will be larger. Also, the frictive pressure will change the bubble wall shape. Since most of the friction is on the symmetric phase side of the wall, the wall will become narrower, see [24], which also increases the friction. Finally, in extensions to the standard model (which are the only viable candidates for baryogenesis because they can provide both a strong phase transition and the heavy Higgs boson required by experiment), the bubble wall is typically thinner, because the Higgs mass is larger. This increases the friction, even before considering new contributions from extra light bosons such as a light scalar top. For these reasons we anticipate that electroweak bubble wall velocities are quite generally much less than 1 . 


\section{REFERENCES}

[1] V.A. Kuzmin, V.A. Rubakov and M.E. Shaposhnikov, Phys. Lett. B155, 36 (1985).

[2] P. Arnold and L. McLerran, Phys. Rev. D 36, 581 (1987).

[3] K. A. Olive, astro-ph/9903309.

[4] M. B. Gavela, P. Hernandez, J. Orloff, O. Pene and C. Quimbay, Nucl. Phys. B430, 382 (1994) hep-ph/9406289.

[5] K. Kajantie, M. Laine, K. Rummukainen and M. Shaposhnikov, Nucl. Phys. B466, 189 (1996) hep-lat/9510020; Phys. Rev. Lett. 77, 2887 (1996) hep-ph/9605288.

[6] M. Laine and K. Rummukainen, Nucl. Phys. B535, 423 (1998) hep-lat/9804019.

[7] P. Arnold, D. Son and L.G. Yaffe, Phys. Rev. D55, 6264 (1997) hep-ph/9609481.

[8] G. D. Moore, Phys. Rev. D59, 014503 (1999) hep-ph/9805264.

[9] D. Bödeker, Phys. Lett. B 426, 351 (1998) hep-ph/9801430.

[10] G.D. Moore, MCGILL/98-28 hep-ph/9810313.

[11] D. Bodeker, G. D. Moore and K. Rummukainen, hep-ph/9907545.

[12] P. Arnold and L. G. Yaffe, hep-ph/9912305.

[13] See for instance, A. E. Nelson, D. B. Kaplan and A. G. Cohen, Nucl. Phys. B373, 453 (1992); Phys. Lett. B336, 41 (1994) hep-ph/9406345; M. Joyce, T. Prokopec and N. Turok, Phys. Rev. D53, 2930 (1996) hep-ph/9410281]; Phys. Rev. D53, 2958 (1996) hep-ph/9410282; J. M. Cline, K. Kainulainen and A. P. Vischer, Phys. Rev. D54, 2451 (1996) hep-ph/9506284; J. M. Cline, M. Joyce and K. Kainulainen, Phys. Lett. B417, 79 (1998) hep-ph/9708393; M. Carena, M. Quiros, A. Riotto, I. Vilja and C. E. Wagner, Nucl. Phys. B503, 387 (1997) hep-ph/9702409.

[14] K. Enqvist, J. Ignatius, K. Kajantie and K. Rummukainen, Phys. Rev. D45, 3415 (1992); J. Ignatius, K. Kajantie, H. Kurki-Suonio and M. Laine, Phys. Rev. D49, 3854 (1994) astro-ph/9309059.

[15] H. Kurki-Suonio and M. Laine, Phys. Rev. D51, 5431 (1995) hep-ph/9501216.

[16] A. F. Heckler, Phys. Rev. D51, 405 (1995) astro-ph/9407064.

[17] P. Huet, K. Kajantie, R. G. Leigh, B. Liu and L. McLerran, Phys. Rev. D48, 2477 (1993) hep-ph/9212224.

[18] H. Kurki-Suonio and M. Laine, Phys. Rev. Lett. 77, 3951 (1996) hep-ph/9607382.

[19] N. Turok, Phys. Rev. Lett. 68, 1803 (1992).

[20] M. Dine, R. G. Leigh, P. Huet, A. Linde and D. Linde, Phys. Rev. D46, 550 (1992) hep-ph/9203203.

[21] B. Liu, L. McLerran and N. Turok, Phys. Rev. D46, 2668 (1992).

[22] S. Y. Khlebnikov, Phys. Rev. D46, 3223 (1992).

[23] P. Arnold, Phys. Rev. D48, 1539 (1993) hep-ph/9302258.

[24] G. D. Moore and T. Prokopec, Phys. Rev. D52, 7182 (1995) hep-ph/9506475.

[25] G. D. Moore and N. Turok, Phys. Rev. D55, 6538 (1997) hep-ph/9608350.

[26] P. Huet and D.T. Son, Phys. Lett. B393, 94 (1997) hep-ph/9610259.

[27] D.T. Son, UW/PT-97-19 hep-ph/9707351.

[28] D. Bodeker, Nucl. Phys. B559, 502 (1999) hep-ph/9905239; hep-ph/9903478.

[29] P. Arnold, D. T. Son and L. G. Yaffe, Phys. Rev. D59, 105020 (1999); Phys. Rev. D60, 025007 (1999) hep-ph/9901304. 